UCRL-JC-131480

PREPRINT

\title{
Bulk Defect Analysis with a High-Energy Positron Beam
}

\author{
J. H. Hartley, R. H. Howell, P. A. Sterne
}

This paper was prepared for submittal to the

Conference on Applications of Accelerators in Research and Industry

Denton, TX

November 4-7, 1998

September 23, 1998

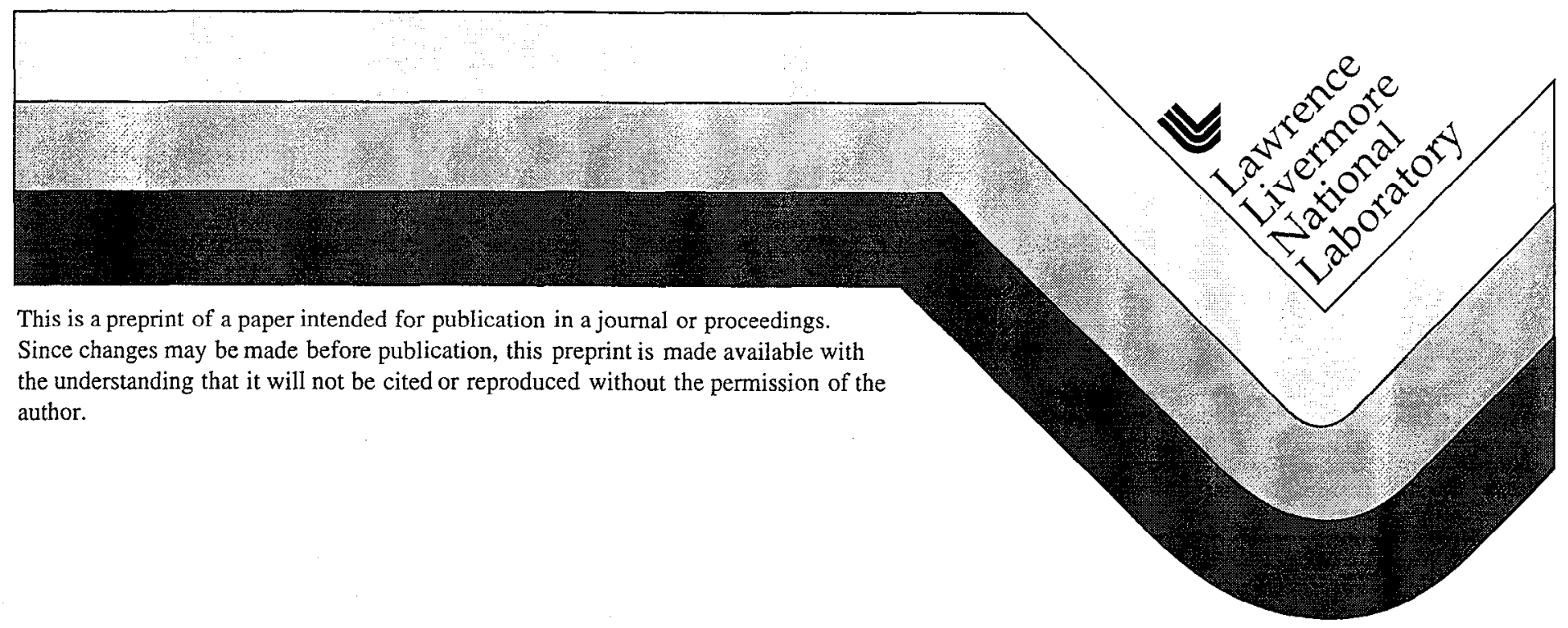




\section{DISCLAIMER}

This document was prepared as an account of work sponsored by an agency of the United States Government. Neither the United States Government nor the University of California nor any of their employees, makes any warranty, express or implied, or assumes any legal liability or responsibility for the accuracy, completeness, or usefulness of any information, apparatus, product, or process

disclosed, or represents that its use would not infringe privately owned rights. Reference herein to any specific commercial product, process, or service by trade name, trademark, manufacturer, or otherwise, does not necessarily constitute or imply its endorsement, recommendation, or favoring by the United States Government or the University of California. The views and opinions of authors expressed herein do not necessarily state or reflect those of the United States Government or the University of California, and shall not be used for advertising or product endorsement purposes. 


\title{
Bulk Defect Analysis with a High-Energy Positron Beam
}

\author{
J. H. Hartley, R. H. Howell, P.A. Sterne \\ Lawrence Livermore National Laboratory, Livermore, CA 94551
}

\begin{abstract}
A program using a positron beam to probe defects in bulk materials has been developed at Lawrence Livermore National Laboratory. Positron annihilation lifetime spectroscopy (PALS) provides non-destructive analysis of average defect size and concentration. A $3 \mathrm{MeV}$ positron beam is supplied by Sodium-22 at the terminal of a Pelletron accelerator. The high-energy beam allows large $\left(\geq 1 \mathrm{~cm}^{2}\right)$ engineering samples to be measured in air or even sealed in an independent environment. A description of the beam-PALS system will be presented along with a summary of recent measurements.
\end{abstract}

\section{INTRODUCTION}

Positron annihilation lifetime spectroscopy is a powerful tool for the study of defects in materials, but it's application has been limited by low data rates and restrictive sample geometry. Samples are sandwiched tightly around a ${ }^{22} \mathrm{Na}$ source, and timing of the positron emission depends on detection of the coincident 1.275 $\mathrm{MeV}$ gamma ray. Positron annihilation in the source results in a $\sim 10 \%$ systematic contribution to the spectrum that complicates the data analysis.

These difficulties can be greatly reduced by performing PALS measurements with a monoenergetic beam. Sample size and geometry are limited only by the beam size and detector configuration. Data rates are significantly improved because positron implantation can be detected with nearly $100 \%$ efficiency. Systematic contributions resulting from positron annihilation in the source are eliminated. In addition, the relatively small phase space of a beam allows better determination of the positron implantation profile.

High-energy positron beams can pass through thin windows before implanting deeply into the sample. This allows in situ measurements of engineering samples at temperature, under stress, or in a controlled atmosphere. The bulk PALS measurements are also insensitive to surface conditions, so no special sample preparation is required.

\section{APPARATUS}

There are both low and high energy positron beams available at LLNL. New developments in our intense, lowenergy beam are found in an accompanying article in this conference.(1) The high-energy beam is derived from a $50 \mathrm{mCi}{ }^{22} \mathrm{Na}$ source moderated by a $1 \mu \mathrm{m}$ thick tungsten single-crystal foil positioned in the terminal of a $3 \mathrm{MV}$ Pelletron electrostatic accelerator. (See Figure 1.) Approximately $1.510^{5} \mathrm{e}^{+} \mathrm{s}^{-1}$ are emitted from the moderator foil. An additional $410^{5} \mathrm{e}^{+} \mathrm{s}^{-1}$ pass through the foil without thermalizing and are picked up in the accelerating field. Both beam components can be focused by a magnetic lens to a $3 \mathrm{~mm}$ spot at a location $2 \mathrm{~m}$ from the exit of the Pelletron for use in PALS. A bending magnet located after the magnetic lens can direct the

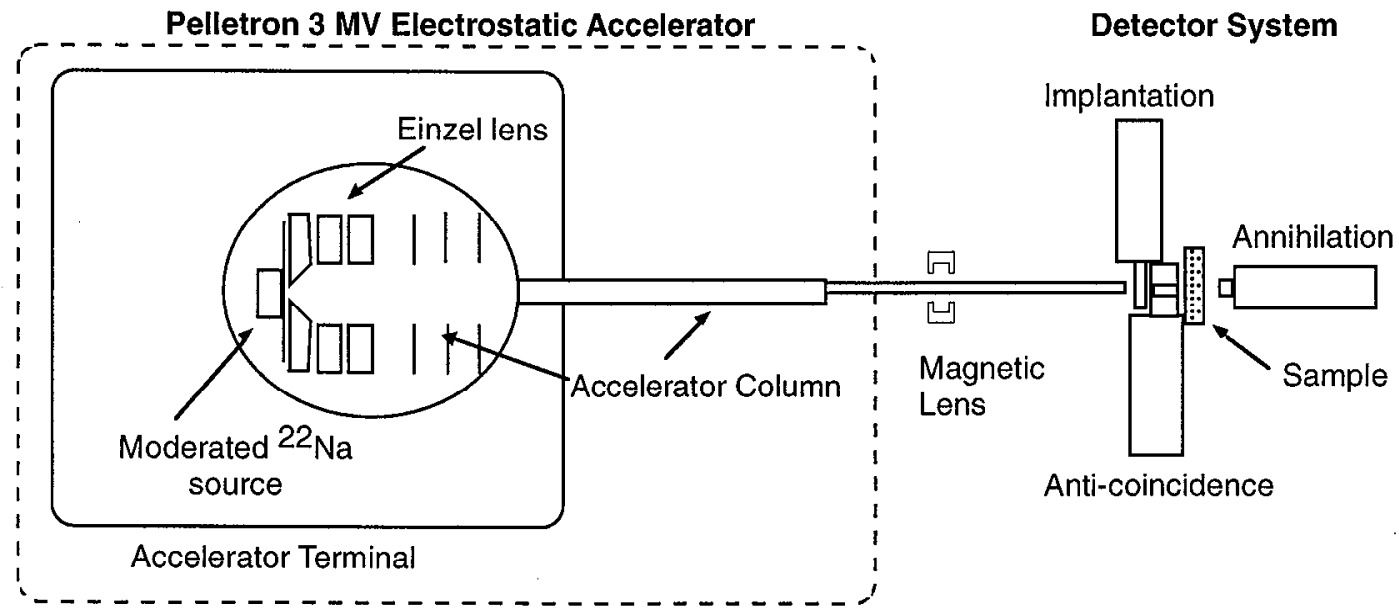

FIGURE 1. Schematic diagram of the high-energy beam-PALS system. 
moderated beam to other experimental stations.

The basic beam-PALS system utilizes three detectors. Positrons exit the accelerator vacuum system through a $20 \mu \mathrm{m}$ aluminum window and pass through a $2 \mathrm{~mm}$ plastic scintillator that measures the positron implantation time. Positrons lose an average of $\sim 200 \mathrm{keV}$ in the detector, and exit in a diverging beam with a broadened energy distribution. The beam diameter is $1 \mathrm{~cm}$ at a sample $4 \mathrm{~cm}$ from the detector.

Positrons implant from $\mathrm{mm}$ to $\mathrm{cm}$ into the sample, depending on its density, thermalize, and annihilate with electrons. Annihilation time is determined from the resulting gamma-rays detected by a $\mathrm{BaF}_{2}$ detector. Time resolution for this spectrometer is generally less than 250 ps even with broad implantation profiles in large, lowdensity samples.

Events from positrons that scatter at large angles in the implantation detector or back-scatter out of the sample are rejected by an anti-coincidence detector positioned as an active collimator. The detector can be constructed with a range of apertures to limit the effective beam diameter on a sample. The smallest beam tested to date was $5 \mathrm{~mm}$ in diameter and gave no increase in the systematic component of the lifetime spectra over the full-size beam.

This three-detector system is a highly efficient lifetime spectrometer with reduced accidental backgrounds and minimal systematic contributions. In the geometry shown in Fig. 1, 2000 counts per second can be achieved with a $1 \mathrm{~cm}$ diameter beam. A systematic contribution appears in the lifetimc spectra at an intensity of $<0.5 \%$. This is attributable to positrons annihilating in the detector system without being seen by the anti-coincidence detector.

Even this systematic can be eliminated by adding a fourth detector, usually $\mathrm{NaI}$, in coincidence with the $\mathrm{BaF}_{2}$ annihilation detector. This geometry reduces the counting rate to less than 800 counts per second but restricts counting to annihilation events in the region between the two detectors. This system is modeled on the one used successfully at Stuttgart Germany.(2)

The four-detector system also allows the use of nonstopping samples. Positrons which pass through the thin sample annihilate more than a meter beyond the coincidence region. The only penalty is a corresponding decrease in the data rate. In low-density samples, the decline in count rate can be balanced against the improvement in timing resolution due to smaller implantation volume.

\section{EXPERIMENTS}

The Pelletron beam is being used to perform basic research as well as non-destructive testing of materials. Basic positron annihilation, channeling and transport properties at high energies are studied in single crystals.(3) A collection of annealed, high-purity metal PALS samples has been assembled with single lifetimes from less than 100 ps to over 200 ps. These will serve as standards for

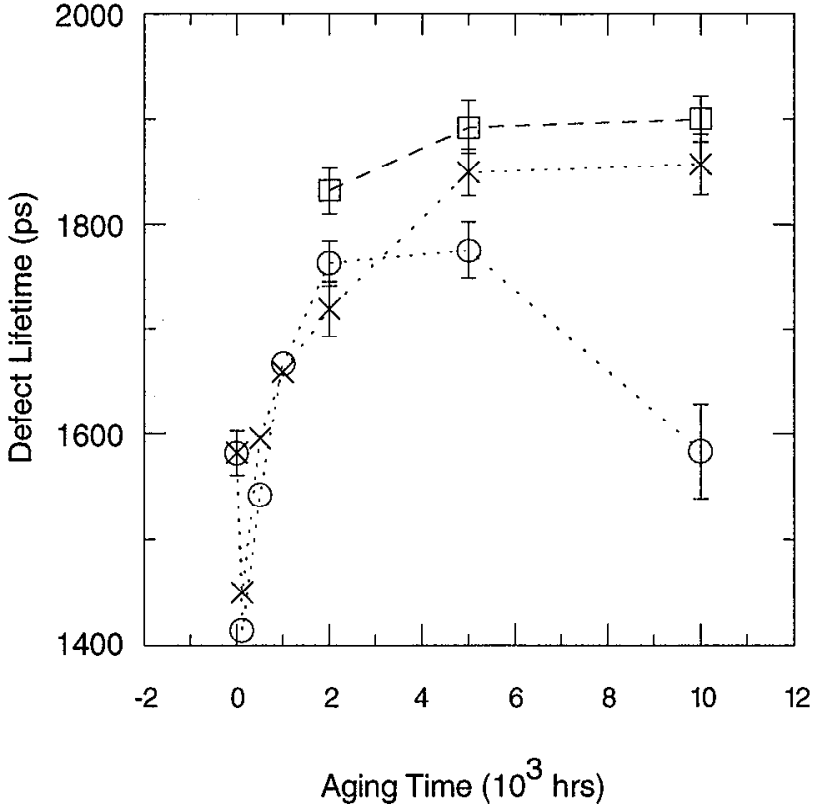

FIGURE 2. Aging of 1908 composite at $200^{\circ} \mathrm{C}$ in argon ( $\square$ ), air $(\mathrm{X})$, and 80/20 argon/nitrogen $(\mathrm{O})$.

comparison to theoretical calculations and testing of data analysis programs.

We are using the high-energy beam-PALS system to study aging effects in carbon fiber resin composites. (See Fig. 2) The composite aging is accelerated by maintaining the samples at elevated temperature in atmospheres of air, argon, or an 80/20 argon-nitrogen mixture.

The lifetime spectra are fit well by two components. The short lifetime $\left(\tau_{2}=370-380 \mathrm{ps}\right)$ is attributed to positrons annihilating in the bulk, and the long component $\left(\mathrm{I}_{2}=3-5 \%\right.$, $\tau_{2}=1.4-1.9 \mathrm{~ns}$ ) is ortho-positronium in defects. The length of this ortho-Ps lifetime is directly related to average hole volume in molecular solids.(4) volume after the first heating cycle of $120 \mathrm{hrs,} \mathrm{followed} \mathrm{by}$ a steady increase in hole size that saturates at 2000-5000 hrs. The sample aged in $80 / 20$ atmosphere for $10,000 \mathrm{hrs}$ began to physically disintegrate, so it's not clear that the measured lifetime decrease is physically meaningful. The hole volume changes are being correlated with infrared spectroscopy and mechanical tests to provide a complete description of the changes during aging.

We are also using the high-energy beam to study metal alloys, actinide metals, and polymeric and molecular solids which have been subjected to irradiation, mechanical stress, and/or chemical degradation. The simple geometry and low dose rate allow us to non-destructively evaluate the same samples used in mechanical tests.

\section{ACKNOWLEDGMENTS}

We'd like to thank Scott Groves for the aged carbon composite samples, and Alan Hunt and William Patterson 
for their assistance in operating and maintaining the Pelletron.

Work performed under the auspices of the U.S. Department of Energy by Lawrence Livermore National Laboratory under Contract No. W-7405-ENG-48.

\section{REFERENCES}

1. P. Asoka-Kumar, R. H. Howell, W. Stoeffl, High-Intensity Positron Program at $L L N L$, Contribution to this proceedings (1998).

2. W. Bauer, J. Briggmann, H.-D. Carstanjen, S. H. Connell, W. Decker, J. Diehl, K. Maier, J. Major, H. E. Schaefer, A. Seeger, H. Stoll and E. Widmann, Nucl. Inst. and Meth. B50, $300(1990)$.

3. L.V. Hau, A.W. Hunt, J. A. Golovchenko, R. Haakenaasen, and K. G. Lynn, Materials Science Forum, 255-257, 119123 (1997).

4. Y. C. Jean, in Positron Spectroscopy of Solids, A. Dupasquier and A. P. Mills, eds. (IOS Press, Amsterdam, 1995), pp 563580. 